\title{
Angiopoiesis and bone regeneration via co- expression of the hVEGF and hBMP genes from an adeno-associated viral vector in vitro and in vivo
}

\author{
Chen ZHANG ${ }^{1}$, Kun-zheng WANG ${ }^{1}$, Hui QIANG ${ }^{1}$, Yi-lun TANG ${ }^{1}$, Qian LI $^{2}$, Miao LI $^{2}$, Xiao-qian DANG ${ }^{1, *}$ \\ ${ }^{1}$ Department of Orthopedic Surgery, ${ }^{2}$ Department of Ultrasound, the Second Affiliated Hospital of Xi'an Jiaotong University, Xi'an \\ 710004, China
}

\begin{abstract}
Aim: To investigate the therapeutic potential of adeno-associated virus (AAV)-mediated expression of vascular endothelial growth factor (VEGF) and bone morphogenetic protein (BMP).

Methods: Four experimental groups were administered the following AAV vector constructs: rAAV-hVEGF 165 -internal ribosome entry site (IRES)-hBMP-7 (AAV-VEGF/BMP), rAAV-hVEGF ${ }_{165}$-GFP (AAV-VEGF), rAAV-hBMP-7-GFP (AAV-BMP), and rAAV-IRES-GFP (AAV-GFP). VEGF 165 and BMP-7 gene expression was detected using RT-PCR. The VEGF 165 and BMP-7 protein expression was determined by Western blotting and ELISA. The rabbit ischemic hind limb model was adopted and rAAV was administered intramuscularly into the ischemic limb. Results: Rabbit bone marrow-derived mesenchymal stem cells (BMSCs) were cultured and infected with the four viral vectors. The expression of GFP increased from the 7th day of infection and could be detected on the 28th day post-infection. In the AAV-VEGF/BMP group, the levels of VEGF165 and BMP-7 increased with prolonged infection time. The VEGF 165 and BMP-7 secreted from BMSCs in the AAV-VEGF/BMP group enhanced HUVEC tube formation and resulted in a stronger osteogenic ability, respectively. In rabbit ischemic hind limb model, GFP expression increased from the 4th week and could be detected at 8 weeks post-injection. The rAAV vector had superior gene expressing activity. Eight weeks after gene transfer, the mean blood flow was significantly higher in the AAV-VEGF/BMP group. Orthotopic ossification was radiographically evident, and capillary growth and calcium deposits were obvious in this group. Conclusion: AAV-mediated VEGF and BMP gene transfer stimulates angiogenesis and bone regeneration and may be a new therapeutic technique for the treatment of avascular necrosis of the femoral head (ANFH).
\end{abstract}

Keywords: adeno-associated virus; vascular endothelial growth factor; bone morphogenetic protein (BMP); avascular necrosis of the femoral head (ANFH); gene therapy

Acta Pharmacologica Sinica (2010) 31: 821-830; doi: 10.1038/aps.2010.67; published online 28 June 2010

\section{Introduction}

Recent insight into the pathogenesis of avascular necrosis of the femoral head (ANFH) has not identified satisfactory methods to increase blood circulation in necrotic areas of the femoral head, to promote bone regeneration, or to prevent osteonecrosis. The rapid development of gene therapy technology is increasingly recognized as a new therapeutic option for the treatment of ANFH, especially through therapeutic neovascularization and bone formation. Among growth factors, vascular endothelial growth factor (VEGF) and bone morphogenetic protein (BMP) play important roles and have been extensively studied.

\footnotetext{
* To whom correspondence should be addressed.

E-mail dang_xiaoqian@sohu.com

Received 2010-02-22 Accepted 2010-05-06
}

The VEGF family of growth factors is one of the most important cytokine families involved in angiogenesis. These factors promote the division of vascular endothelial cells and induce angiopoiesis. VEGF growth factors are essential for bone formation and repair during the bone regeneration process, which directly attracts endothelial cells and osteoclasts and enhances the differentiation of osteoblasts ${ }^{[1,2]}$. BMP growth factors are the only signaling molecules that are individually sufficient for the induction of bone formation at orthotopic and heterotopic sites. They have defined roles in stimulating the proliferation and differentiation of mesenchymal and osteoprogenitor cells and have efficient bone induction activity ${ }^{[3,4]}$. Because bone formation is a coordinated process involving the BMP and VEGF growth factors ${ }^{[5,6]}$, orchestrating the timing with which these two factors are expressed may greatly enhance this process. 
Choosing a safe and effective vector system to transfer and correctly express a target gene during gene therapy is important. Several different strategies have been examined for the delivery of genes of interest, including the use of naked DNA or an adenoviral vector. Treatment with naked DNA is simple and well tolerated by the recipient organism due to its low toxicity and weak induction of immune responses. However, the transduction efficiency is significantly lower when compared with other methods. The adenovirus has frequently been the vector of choice for gene transfer because it is able to transduce a variety of cells with high efficiency. However, adenoviral vectors have major limitations, including a lack of sustained expression, the antigenicity of viral proteins that are targeted by both humoral immunity and cytotoxic $\mathrm{T}$ lymphocytes, and possible toxicity at high doses. However, there are many inherent features of the adeno-associated virus system that make it an attractive option as a human viral vector. AAV is a non-pathogenic, defective human parvovirus that requires the presence of a helper virus, such as adenovirus or herpes virus, for productive infection ${ }^{[7,8]}$. Other advantages of this vector system include its low immunogenicity, its ability to transduce both dividing and non-dividing cells, the potential to integrate into specific sites, its ability to achieve long-term gene expression (even in vivo), and its broad tropism, allowing for the efficient transduction of diverse organs ${ }^{[9]}$. These features make AAV attractive and efficient for gene transfer in vitro and local injection in vivo.

To enhance neovascularization and bone regeneration during osteonecrosis therapy, we constructed adeno-associated viruses co-expressing hVEGF $_{165}$ and hBMP-7 (rAAV-VEGF ${ }_{165}-$ IRES-BMP-7) and detected their effect on gene expression and biological activity in vitro and in vivo. These data demonstrate the synergistic action of these two genes and may provide a new therapeutic option for ANFH.

\section{Materials and methods Materials and reagents}

The rAAV-hVEGF ${ }_{165}$-IRES-hBMP-7 (AAV-VEGF/BMP), rAAVhVEGF ${ }_{165}$-GFP (AAV-VEGF), rAAV-hBMP-7-GFP (AAV-BMP), and rAAV-IRES-GFP (AAV-GFP) plasmids were constructed by Dr Xiang-hui HUANG. Human embryonic kidney cells293 (HEK-293) and human umbilical vein endothelial cells (HUVECs) were obtained from the Department of Orthopedic Surgery in the Second Affiliated Hospital of Xi' an Jiaotong University. Male New Zealand rabbits (two months old, weighing $2.0-3.0 \mathrm{~kg}$ ) were obtained from the experimental animal center of $\mathrm{Xi}^{\prime}$ an Jiao Tong University. All animal protocols followed the recommendations and guidelines of the National Institutes of Health and were approved by the $\mathrm{Xi}^{\prime}$ an Jiao Tong University Animal Care and Use Committee. The AAV helper-free system was obtained from Stratagene (La Jolla, CA, USA). A schematic representation of the structure of the plasmids in the AAV Helper Free System is provided in Figure 1A.
A
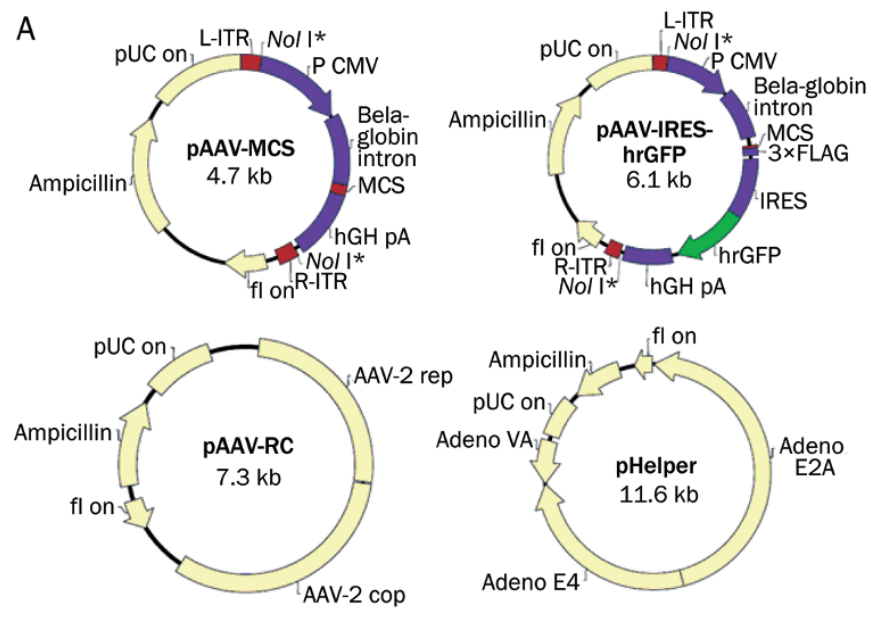

B
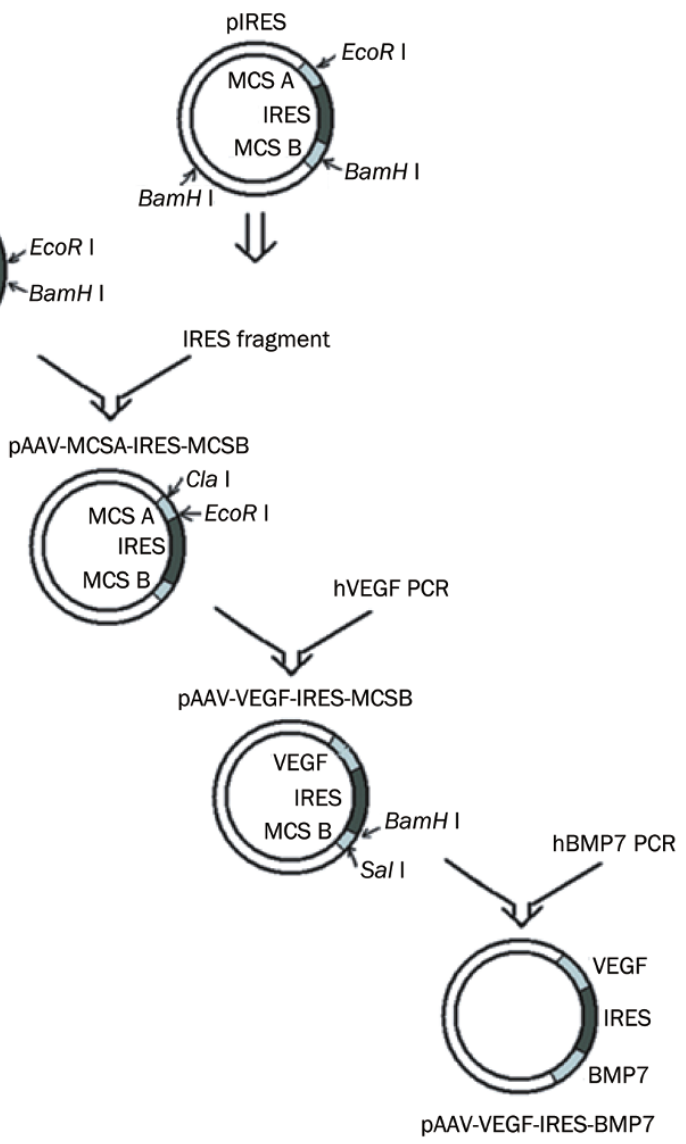

Figure 1. (A) Schematic representation of the structure of plasmids in AAV Helper Free System. (B) Conceptual diagram of construction of pAAVhVEGF $_{165}$-IRES-hBMP-7. hVEGF 165 gene $(600 \mathrm{bp})$ and hBMP-7 gene (1300 bp) were respectively inserted into upstream MCS and downstream MCS located on either side of IRES sequence (631 bp). The length of the bicistronic frame is $2.5 \mathrm{~kb}$.

\section{rAAV vector production}

The construction of the rAAV-hVEGF 165 -IRES-hBMP-7 (AAVVEGF/BMP), rAAV-hVEGF ${ }_{165}$-GFP (AAV-VEGF), rAAV- 
hBMP-7-GFP (AAV-BMP), and rAAV-IRES-GFP (AAV-GFP) vectors was carried out as previously described ${ }^{[10]}$. The structure of the pAAV-hVEGF ${ }_{165}$-IRES-hBMP-7 vector is shown in Figure 1B. IRES sequences were incorporated into the pAAV MCS to construct a bicistronic vector with two multiple cloning sites. Then, the $\mathrm{hVEGF}_{165}$ (Pubmed NM-003376) and hBMP-7 (Pubmed NM-001719) genes were inserted into the upstream and downstream MCS, respectively. The length of the bicistronic frame is $2.5 \mathrm{~kb}$, which is within the capacity of the vector. The AAV helper-free system was used to generate recombinant AAV. HEK-293 cells were cultured in H-DMEM supplemented with $10 \%$ fetal bovine serum containing 20 $\mathrm{mg} / \mathrm{mL}$ penicillin-streptomycin and incubated with $5 \% \mathrm{CO}_{2}$ at $37^{\circ} \mathrm{C}$. The AAV vector was co-transfected with the pAAVhelper and pAAV-RC vectors into HEK 293 cells by a calcium phosphate method according to the manufacturer's instructions (Invitrogen, Carlsbad, CA, USA). A primary virus stock was collected $72 \mathrm{~h}$ after transfection and further concentrated and purified by chloroform/PEG8000 protocols ${ }^{[11]}$. The recombinant adeno-associated virus had a titer of $5.5 \times 10^{11} \mathrm{vp} / \mathrm{mL}$.

\section{Rabbit bone marrow-derived mesenchymal stem cells (BMSC)} culture and rAAV infection in vitro

Male New Zealand rabbits were used to obtain rabbit BMSCs. The cells were harvested by gently flushing the tibiae and femora with L-DMEM. Density gradient centrifugation and adherent screening methods were used to isolate BMSCs as previously described ${ }^{[12]}$. The cells were cultured in L-DMEM supplemented with $10 \%$ fetal bovine serum containing 20 $\mathrm{mg} / \mathrm{mL}$ penicillin-streptomycin and incubated with $5 \% \mathrm{CO}_{2}$ at $37^{\circ} \mathrm{C}$. Following the $3 \mathrm{rd}$ passage, BMSCs $\left(5 \times 10^{4}\right.$ cells/well $)$ were seeded onto 24 -well plates $24 \mathrm{~h}$ before rAAV infection. By taking into account the cytopathogenic effect, infection efficiency, and cost of recombinant virus, we determined that the best multiplication of infection (MOI) for infecting rabbit BMSCs with rAAV was $5 \times 10^{4} \mathrm{vp} /$ cell. The four rAAV virus variants were introduced into BMSCs using this MOI. Cells were incubated as above and were swirled gently at 30-min intervals. One hour later, the medium was replaced with $L$-DMEM supplemented with $10 \%$ fetal bovine serum. Medium was then completely replaced every three days.

\section{Rabbit hind limb ischemia model and rAAV infection in vivo}

Male New Zealand rabbits were kept under specific pathogen free conditions and supplied with sterile food and acidified water. The hind limb ischemia model was developed as described previously ${ }^{[13]}$. Rabbits were anesthetized with an intraperitoneal injection of sodium pentobarbital $(50 \mathrm{mg} / \mathrm{kg})$. Under a surgical microscope, a vertical longitudinal incision was made in the right hind limb. The right femoral arteries were separated from the origin of the external iliac artery, ligated, and completely excised. Immediately after ligation of the femoral artery, the four rAAV virus variants were each injected into five different $\operatorname{sites}^{[14]}$ on the three major thigh muscles of each rabbit $\left(5.5 \times 10^{11} \mathrm{vp} / 20 \mu \mathrm{L}\right.$ per site), including the adductor (two sites), the quadriceps (two sites), and the semimembranous (one site) muscles. Subsequently, the skin was sutured. After surgery, all animals were housed under standard conditions (temperature: $21 \pm 1{ }^{\circ} \mathrm{C}$; humidity: $55 \%-$ $60 \%$ ) with food and water continuously available. The hind limbs were mobilized without any fixation. To prevent infection, animals received prophylactic injections of gentamicin $\left(0.03 \mathrm{mg} \cdot \mathrm{kg}^{-1} \cdot \mathrm{d}^{-1}, \mathrm{im}\right)$ within 3 days after surgery.

Rabbits were sacrificed at various time points post-injection to characterize gene expression efficiency and the effects on angiopoiesis and bone regeneration in vivo. Each group contained 30 rabbits and was divided into four experimental subgroups: group A $(n=6)$ was examined at week 2 for GFP expression $(n=3)$ and immunoblotting $(n=3)$, group $\mathrm{B}(n=6)$ at week 4 for GFP expression $(n=3)$ and immunoblotting $(n=3)$, group C $(n=9)$ at week 6 for GFP expression $(n=3)$, immunoblotting $(n=3)$, and ELISA $(n=3)$, and group D $(n=9)$ at week 8 for GFP expression $(n=3)$ and for blood flow measurement, X-ray radiography, and immunohistochemistry $(n=6)$.

\section{Reporter gene (GFP) expression in vitro and in vivo}

Following 3, 7, 14, and 28 days of infection with AAV-GFP virus in vitro, the expression of GFP protein was observed by inverted fluorescence microscopy. At 2, 4, 6, and 8 weeks post-injection in vivo, the muscles injected with the AAV-GFP virus were sliced by the frozen section method and the expression of the GFP protein was observed as above. Each assay was performed in triplicate.

Preparation of culture medium and assessment of VEGF $_{165}$ and BMP-7 gene expression

Total cellular RNA was isolated at 1, 2, 3, 7, 14, 21, and 28 days following infection with the AAV-GFP, AAV-VEGF, AAV-BMP or AAV-VEGF/BMP viruses using TRIzol Reagent (Invitrogen). Extracted RNA was treated with DNase I (Takara, Tokyo, Japan) to eliminate DNA contamination, and first-strand cDNA was synthesized with random hexamer primers using the reverse first-strand cDNA synthesis kit from MBI Fermentas (Glen Burnie, MD, USA). PCR was performed to amplify humanVEGF ${ }_{165}$ (forward primer 5'-CCATCGATATGAACTTTCTGCTGTCTTG-3'; reverse primer 5'-CGGAATTCTCACCGCCTCGGCTTGTC-3') and BMP-7 (forward primer 5'-GGCCGGATCCATGCACGTGCGCTCACTGCG-3'; reverse primer $5^{\prime}$-GGCCGTCGACCTAGTGGCAGCCACAG-3'). $\quad \beta$-actin (forward primer 5'-GAGGGAAATCGTGCGTGAC-3'; reverse primer 5'-TAGGAGCCAGGGCAGTAATCT-3') was detected by RT-PCR as an internal control. PCR was performed using the following program: $94{ }^{\circ} \mathrm{C}$ for 3 min for one cycle and 35 cycles at $94^{\circ} \mathrm{C}$ for $30 \mathrm{~s}, 55^{\circ} \mathrm{C}$ for $30 \mathrm{~s}$, and $72{ }^{\circ} \mathrm{C}$ for $45 \mathrm{~s}$. The PCR products were electrophoresed on ethidium bromide-stained 2.0\% agarose gels. Each assay was performed in triplicate.

Muscle extract preparation and assessment of $\mathrm{VEGF}_{165}$ and BMP7 gene expression

At 2, 4, and 6 weeks following injection with the AAVGFP, AAV-VEGF, AAV-BMP, or AAV-VEGF/BMP viruses, 
the frozen muscles were pulverized in liquid nitrogen and homogenized in $3 \mathrm{~mL}$ of ice-cold lysis buffer $(1 \%$ Nonidet P-40; 50 mmol/L Tris-HCl, pH 7.4; $150 \mathrm{mmol} / \mathrm{L} \mathrm{NaCl} ; 200$ $\mathrm{U} / \mathrm{mL}$ aprotinin; $1 \mathrm{mmol} / \mathrm{L}$ phenylmethylsulfonyl fluoride, PMSF). The tissue lysates (50 $\mathrm{mg}$ of protein) were separated by $12 \%$ polyacrylamide gel electrophoresis and blotted onto polyvinylidene difluoride membranes. Immunoblotting was performed with anti-human VEGF $_{165}$ and BMP-7 antibodies and the specific binding of the antibody was visualized with an ECL detection system. At 6 weeks post-injection, muscle extracts were measured with an enzyme-linked immunosorbent assay (ELISA) kit using the Biotrak ELISA system (R\&D, Minneapolis, MN, USA) according to the manufacturer's instructions. Each assay was performed in triplicate.

\section{Angiogenic and osteogenic in vitro assays Tube formation assay}

HUVECs were cultured as previously described ${ }^{[15]}$. Basement membrane matrigel matrix (BD, Bedford, MA, USA) was diluted by serum-free medium, added to a 24-well plate, and incubated at $37^{\circ} \mathrm{C}$ for $30 \mathrm{~min}$ to allow solidification to occur. HUVECs $\left(5 \times 10^{4}\right.$ cells/well) were seeded on the matrigel and fresh $L$-DMEM medium supplemented with 10\% FBS was added. Next, $1 \mathrm{~mL}$ of culture supernatant was harvested from the AAV-GFP, AAV-VEGF, AAV-BMP, or AAV-VEGF/BMP groups 14 days post-infection and added to the 24-well plate. The plate was then incubated at $37{ }^{\circ} \mathrm{C}$ with $5 \% \mathrm{CO}_{2}$ for $12 \mathrm{~h}$. The images of tube formation were captured under a light microscope from three random fields, and quantification of the tubes was analyzed by image processing software (Media Cybernetics, USA) to assess the biological activity of VEGF in vitro.

\section{Mineralization assay}

BMSCs were infected with the four virus groups above. The cells were then cultured in L-DMEM supplemented with $10 \%$ fetal bovine serum containing $20 \mathrm{mg} / \mathrm{mL}$ penicillinstreptomycin with $5 \% \mathrm{CO}_{2}$ at $37{ }^{\circ} \mathrm{C}$ (the culture medium did not contain osteogenic induction factors, such as ascorbic acid, $\beta$-glycerophosphate, or dexamethasone). Mineralization effects were detected by von Kossa and alizarin red (AZR) staining ${ }^{[16]}$ for calcium deposits 4 weeks post-infection and observed using an inverted phase contrast microscope. The images of mineral nodules were captured under a light microscope from three random fields, and quantification of the mineral nodules was analyzed by image processing software to assess the biological activity of BMP in vitro.

\section{Blood flow measurement and orthotopic bone formation in vivo}

Eight weeks after injection, rabbits in the four groups were anesthetized with an intraperitoneal injection of sodium pentobarbital $(50 \mathrm{mg} / \mathrm{kg}$ ). Blood flow in the anterior tibial artery of ischemic and normal hind limbs was measured at rest with an Aspen Advanced Doppler ultrasound device from Acuson (Siemens Medical Solutions, Mountain View, CA, USA) using a perivascular flow probe and calculated by the inlay automatic processing software. The data were expressed as a percentage of the contralateral limbs. Three separate measurements were performed for each rabbit at every time point and the results were averaged. In addition, rabbits in the four groups were subjected to X-ray radiography to assess orthotopic bone formation.

\section{Histological assessment}

Eight weeks after injection, thigh muscle tissue sections of ischemic limbs from the four groups were harvested and fixed in $10 \%$ neutral-buffered formalin. To identify the proliferation of capillary endothelial cells, tissue sections were immunostained for CD34. The monoclonal antibody against CD34 was applied at a 1:500 dilution after blocking with 1\% normal bovine serum. Subsequent incubation with biotinylated horse anti-mouse IgG and an ABC Elite kit (Santa Cruz) was performed. The number of CD34-positive vessels was counted at a magnification of $200 \times$, and twenty fields from each typical slide were counted (mean number of capillaries per square millimeter). To assess orthotopic bone formation, the slides were stained by von Kossa staining to detect mineralization.

\section{Statistical analysis}

The results are reported as means \pm standard deviation. The normality of the data distribution was assessed with the Shapiro-Wilk (W) test. ANOVA followed by the Fisher's test was conducted to assess differences among treatment groups. Statistical significance was set at a $P$-value less than or equal to 0.05 . The SPSS mathematical statistics software used for this analysis was purchased from SPSS Inc (version 8; SPSS Inc, Chicago, IL, USA).

\section{Results}

Animal condition after rAAV infection

There were no symptoms of local or systemic toxicity after rAAV infection. In the region of the injection sites, no inflammatory reaction, such as rubeosis, engorgement, or abscessus, was observed. The activities of all animals were normal. There was no systemic toxicity, such as nutation, instability of gait, anhelation, retardation, cyanosis, or convulsion. No animals died before the end of the experiments.

\section{GFP gene expression}

In vitro: GFP protein expression could be detected on the third day post-infection. However, the efficiency and density of infection were unstable. The expression of GFP protein increased from the 7 th day and could be detected at 28 days post-infection (Figures 2A, 2B). In vivo: With prolonged infection time, GFP protein expression increased from the 4 th week and could be detected at 8 weeks post-infection (Figures 2C, 2D).

\section{Efficient genes expression of $\mathrm{hVEGF}_{165}$ and $\mathrm{hBMP}-7$}

To confirm hVEGF $_{165}$ and hBMP-7 gene expression in vitro, RT-PCR assays were performed. As shown in Figure $3 \mathrm{~A}-3 \mathrm{D}$, the sizes of the PCR products for $\mathrm{VEGF}_{165}, \mathrm{BMP}-7$, 
A

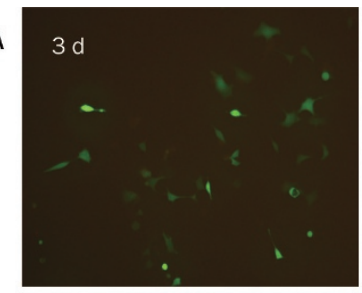

B

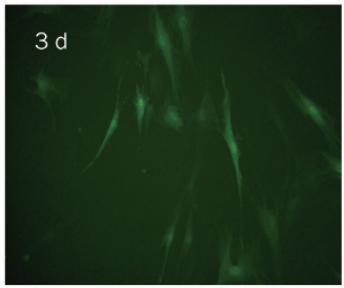

c

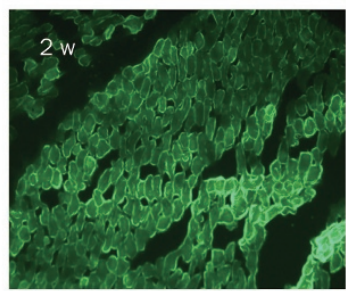

D

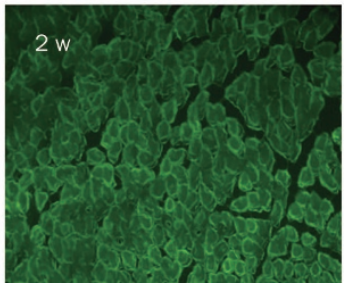

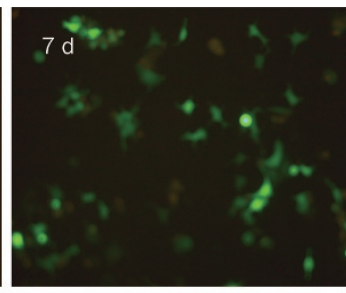
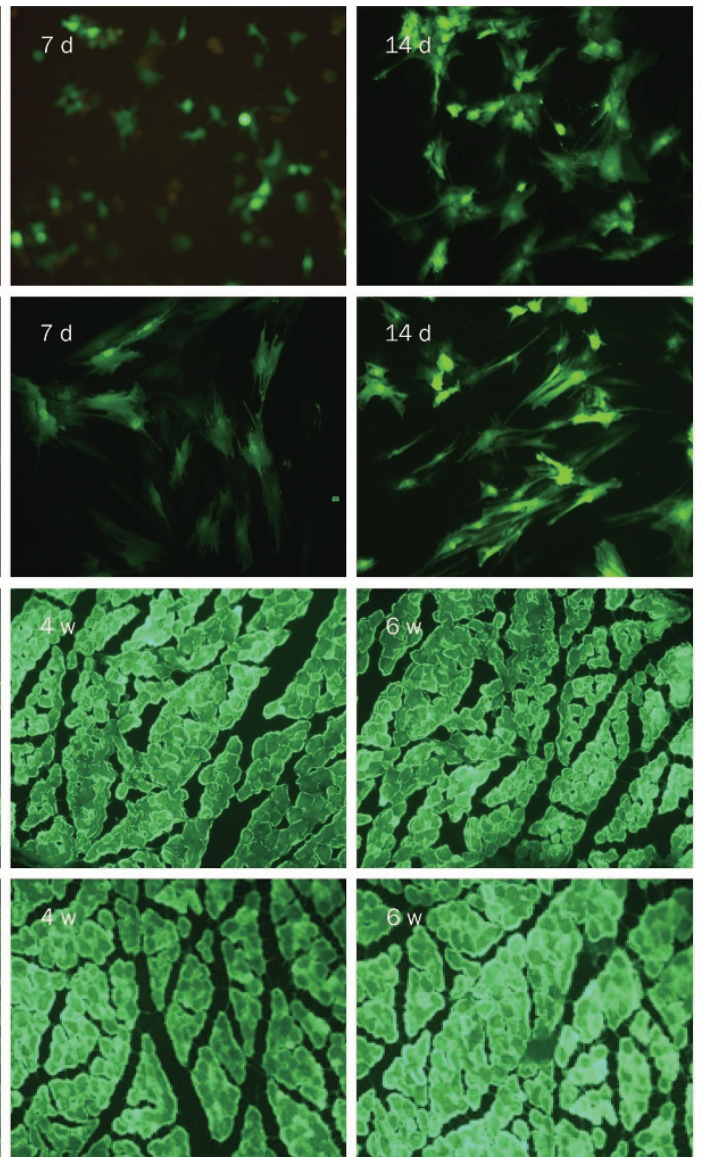

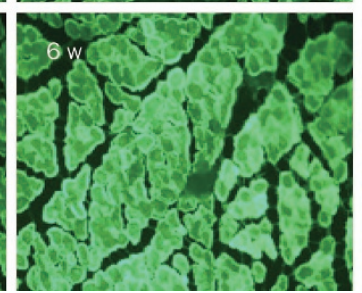

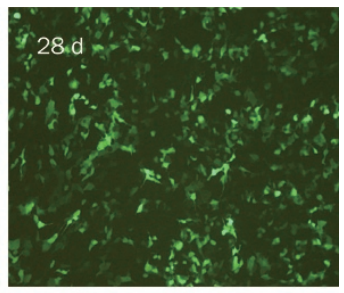
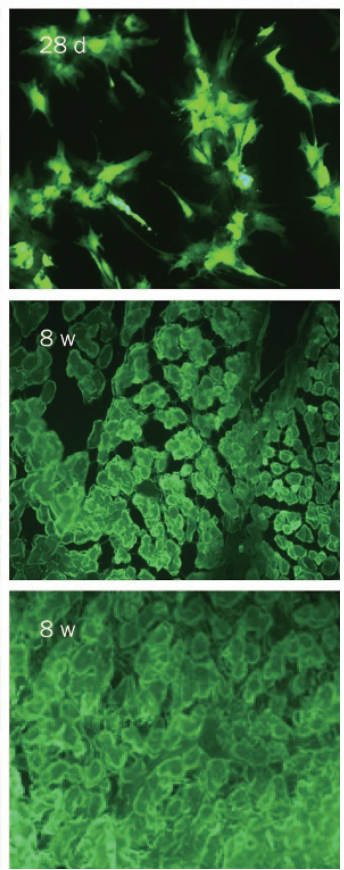

Figure 2. Representative images of GFP protein expression. (A-B) on the 3 rd, 7th, 14th, and 28th days after rAAV-IRES-GFP virus transfection in vitro. (A) Magnification $\times 100$; (B) Magnification $\times 200$; (C-D) on the 2nd, 4th, 6th, and 8th weeks after rAAV-IRESGFP virus injection in vivo. (C) Magnification $\times 100$; (D) magnification $\times 200$. and $\beta$-actin were $600 \mathrm{bp}, 1300 \mathrm{bp}$, and $340 \mathrm{bp}$, respectively. With prolonged infection time, the intensity of the $\mathrm{VEGF}_{165}$ and BMP-7 bands increased in the AAV-VEGF/BMP group. Together, these data demonstrate that $\mathrm{VEGF}_{165}$ was expressed in the AAV-VEGF and AAV-VEGF/BMP groups but not in the AAV-BMP and AAV-GFP groups and that BMP-7 was expressed in the AAV-BMP and AAV-VEGF/BMP groups but not in the AAV-VEGF and AAV-GFP groups. Protein expression of 2, 4, and 6 weeks following injection with AAV-VEGF/ BMPin vivo is shown in Figure 3E-3H . Expression of the VEGF $_{165}$ and BMP-7 proteins was visualized by Western blot analysis. Strong staining at the expected molecular weights of $23 \mathrm{kDa}\left(\mathrm{hVEGF}_{165}\right), 55 \mathrm{kDa}(\mathrm{hBMP}-7)$, and $43 \mathrm{kDa}(\beta$-actin) was observed. With prolonged infection time, the intensity of the $\mathrm{VEGF}_{165}$ and BMP-7 bands increased. These data demonstrate that $\mathrm{VEGF}_{165}$ was expressed in the AAV-VEGF and AAV-VEGF/BMP groups but not in the AAV-BMP and AAVGFP groups and that BMP-7 was expressed in the AAV-BMP and AAV-VEGF/BMP groups but not in the AAV-VEGF and AAV-GFP groups. As shown in Figure 3I, 3J, the production of $\mathrm{hVEGF}_{165}$ and hBMP-7 was quantified in relevant muscle extracts 6 weeks post-injection. The average amounts of hVEGF $_{165}$ protein in the AAV-VEGF/BMP and AAV-VEGF groups were significantly higher than those in the AAV-GFP and AAV-BMP groups $(P<0.05, n=30)$. The average amounts of hBMP-7 protein in the AAV-VEGF/BMP and AAV-BMP groups were significantly higher than those in the AAV-GFP and AAV-VEGF groups $(P<0.05, n=30)$.

\section{Biological activity of $\mathrm{hVEGF}_{165}$ and $\mathrm{hBMP}-7$ in vitro}

As shown in Figure 4A, hVEGF $_{165}$ secreted from BMSCs in the AAV-VEGF/BMP group enhanced HUVEC migration, proliferation, and tube formation in comparison with the other three groups. The number of tubes in the AAV-VEGF/BMP group was significantly higher than that in the AAV-GFP and AAV-BMP groups. However, there was no statistical difference between the AAV-VEGF/BMP group and the AAVVEGF group (Figure 4B). In addition, the mineralization effect of hBMP-7 was detected by von Kossa (Figure 5A) and alizarin red staining (Figure 5B). The AAV-VEGF/BMP group displayed stronger osteogenic activity than all the other groups. The number of mineralized nodules in the AAV-VEGF/BMP group was significantly higher than that in the AAV-GFP and AAV-VEGF groups. However, there was no statistical difference between the AAV-VEGF/BMP group and the AAV-BMP group (Figure 5C).

\section{Biological activity of hVEGF $_{165}$ and hBMP-7 in vivo}

The ability of hVEGF $_{165}$ and hBMP-7 to induce tube formation and mineralization in vitro correlated well with their in vivo role in neovascularization and bone regeneration. Blood flow in the anterior tibial artery of ischemic and normal hind 

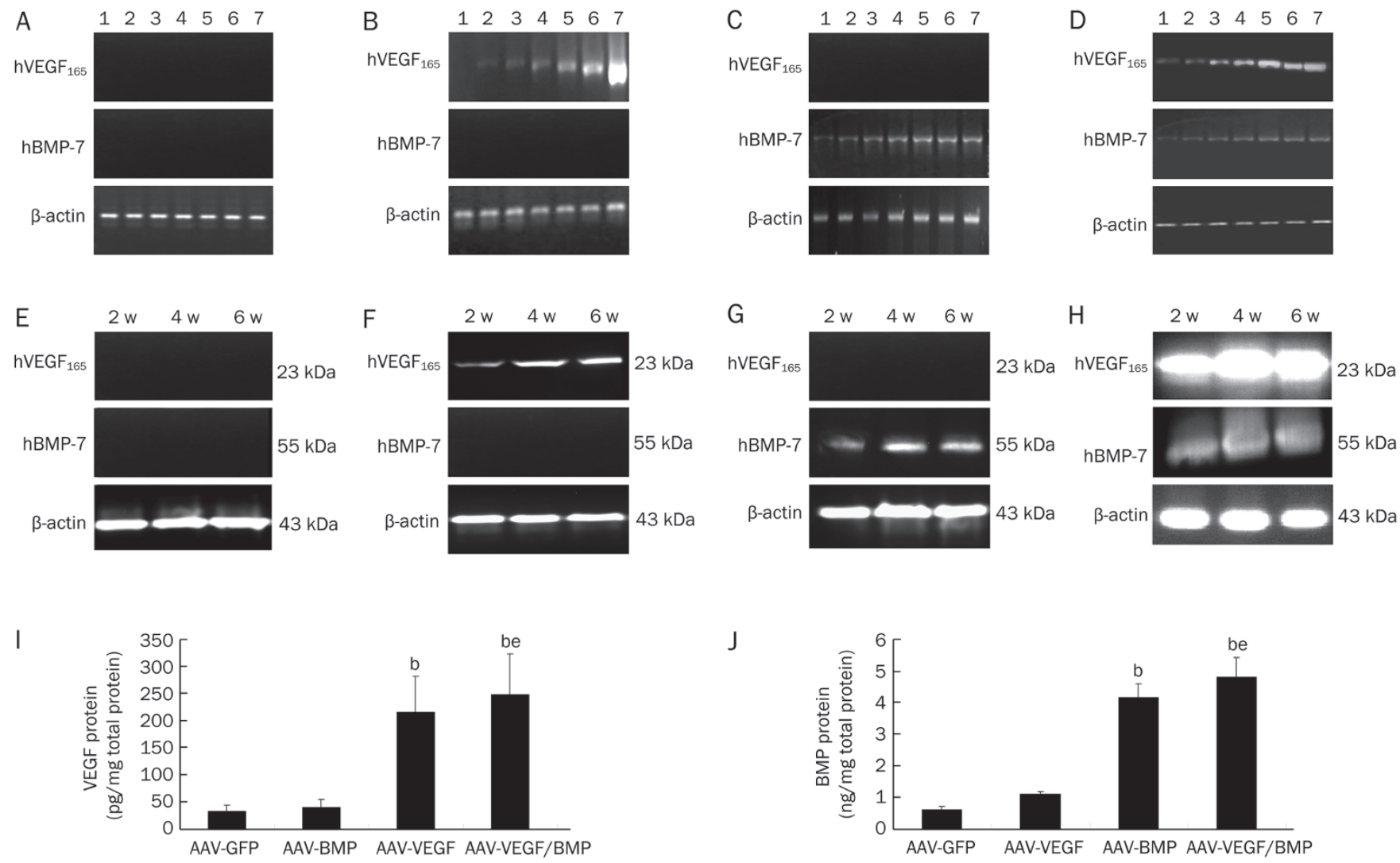

J

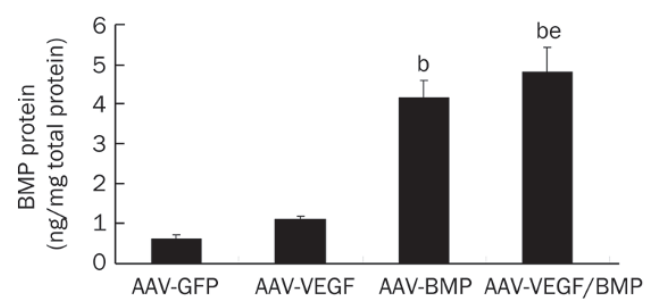

Figure 3. Expression of $\mathrm{hVEGF}_{165}$ and hBMP-7. (A-D) Representative images of RT-PCR assay of AAV-GFP group (A), AAV-VEGF group (B), AAV-BMP group (C)

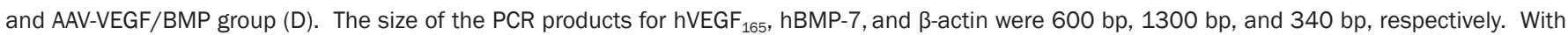
prolonged infection time, the brightness of the VEGF VE5 $_{165}$ or BMP-7 bands increased in AAV-VEGF/BMP group. No hVEGF 165 or hBMP-7 band could be detected in AAV-GFP group. Track 1-7 stands for the 1st, 2nd, 3rd, 7th, 14th, 21st, and 28th days post-transfection. (E-H) Representative images of Western blotting assay of AAV-GFP group (E), AAV-VEGF group (F), AAV-BMP group (G) and AAV-VEGF/BMP group (H). The molecular weights of hVEGF ${ }_{165}$, hBMP-7, and $\beta$-actin were $23 \mathrm{kDa}, 55 \mathrm{kDa}$ and $43 \mathrm{kDa}$ respectively. Strong staining with the expected molecular weight was observed in AAV-VEGF/ BMP group, and no hVEGF 165 or hBMP-7 band was observed in AAV-GFP group. (I) ELISA assay for VEGF protein expression. The data is expressed as the mean $\pm S D$ from three independent experiments. ${ }^{b} P<0.05$ vs AAV-GFP group, ${ }^{e} P<0.05$ vs AAV-BMP group. (J) ELISA assay for BMP protein expression. The data is expressed as the mean \pm SD from three independent experiments. ${ }^{b} P<0.05$ vs AAV-GFP group. ${ }^{e} P<0.05$ vs AAV-VEGF group.

limbs was measured 8 weeks post-injection to assess the neovascularization capability of $\mathrm{hVEGF}_{165}$ in vivo. As shown in Figure 6, the ratio of mean ischemic/normal blood flow in the AAV-VEGF/BMP group was highest when compared with the AAV-GFP, AAV-VEGF, and AAV-BMP groups. However, there was no statistical difference between the AAV-VEGF/ BMP group and the AAV-VEGF group. In addition, rabbits in the four groups were subjected to $\mathrm{X}$-ray radiography to assess the bone regeneration activity of hBMP-7 in vivo. As shown in Figure 7, orthotopic ossification was radiographically evident in the AAV-VEGF/BMP group eight weeks post-injection. In contrast, no radiographic evidence of bone formation was observed in the AAV-GFP group or the AAV-VEGF group.

\section{Histological assessment}

To further assess vascularity in rAAV-infected muscle, immunostaining for CD34, a marker of vessel endothelial cells, was performed to detect the number of capillaries. As shown in Figure 8A, muscle from the AAV-VEGF/BMP group con- tained significantly more capillaries when compared with the other three groups at 8 weeks post-injection. The mean density of capillaries in the AAV-VEGF/BMP group was significantly higher than that in the AAV-GFP and AAV-BMP groups. However, there was no statistical difference between the AAV-VEGF/BMP group and the AAV-VEGF group (Figure $8 \mathrm{C}$ ). To analyze bone formation, von Kossa staining was adopted to assess calcium deposits 8 weeks post-injection. As shown in Figure 8B, calcium deposits stained black. The osteogenic ability of the AAV-VEGF/BMP group was significantly enhanced compared with that found in the other three groups.

\section{Discussion}

The packaging capacity of the rAAV vector $(5 \mathrm{~kb}$, including the inverted terminal repeats) remains one of its primary limitations in terms of gene delivery. However, substantial progress has recently been made to overcome this restriction. Among the several different strategies to co-express multiple 

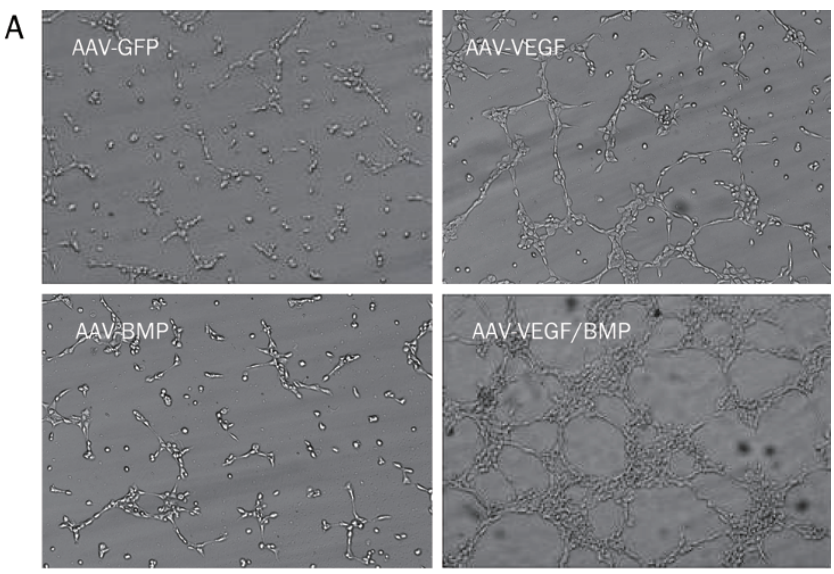

B

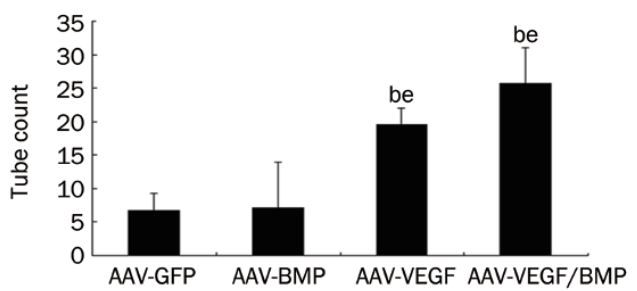

Figure 4. Tube formation experiment with HUVECs. (A) Representative images of tube formation. The HUVECs of AAV-VEGF/BMP group displayed stronger migration, proliferation, and tube formation ability than other three groups (magnification $\times 200$ ). (B) The data is expressed as the mean $\pm S D$ from three independent experiments. ${ }^{b} P<0.05$ vs AAV-GFP group. ${ }^{\mathrm{e}} \mathrm{P}<0.05$ vs AAV-BMP group.

genes, the incorporation of an IRES into this gene therapy vector represents one of the more promising strategies ${ }^{[17-19]}$. The IRES functions as a ribosome landing pad for the efficient internal initiation of translation, ensuring coordinated expression of several genes. The IRES initiates ribosome binding and translation in the absence of a $5^{\prime} \mathrm{CAP}$, thus overcoming the main disadvantage of traditional strategies that express two different genes. This characteristic is especially useful for AAV production due to the packaging size limitation imposed by the AAV vectors. In our current study, an IRES sequence was incorporated into the pAAV MCS to construct a bicistronic vector. Then, the hVEGF 165 and hBMP-7 genes were inserted upstream and downstream of the MCS, located on either side of the IRES to create a bicistronic frame of 2.5 $\mathrm{kb}$ in length, which is within the capacity of the vector. In our study, we reveal that AAV-mediated hVEGF $_{165}$ and hBMP-7 gene transfer in vitro and in vivo induces the expression and secretion of the hVEGF $_{165}$ and hBMP-7 proteins. These results demonstrate that the IRES sequence may be a superior strategy for co-expressing multiple genes in rAAVs.

An important characteristic of rAAV is that when the host cell is infected with rAAV, the efficiency of infection cannot immediately be determined. The expression of the gene of interest will not be activated until the double-stranded nucleic acid version of the virus has been synthesized by DNA syn-
A
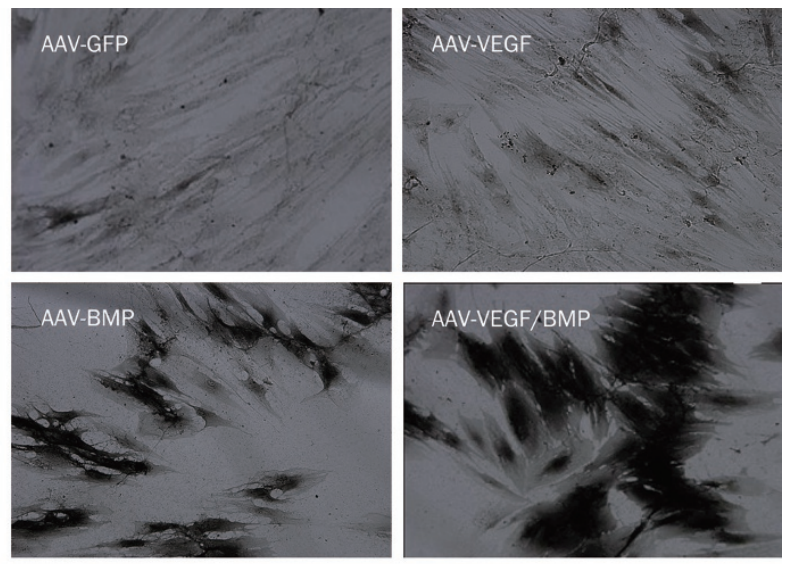

B
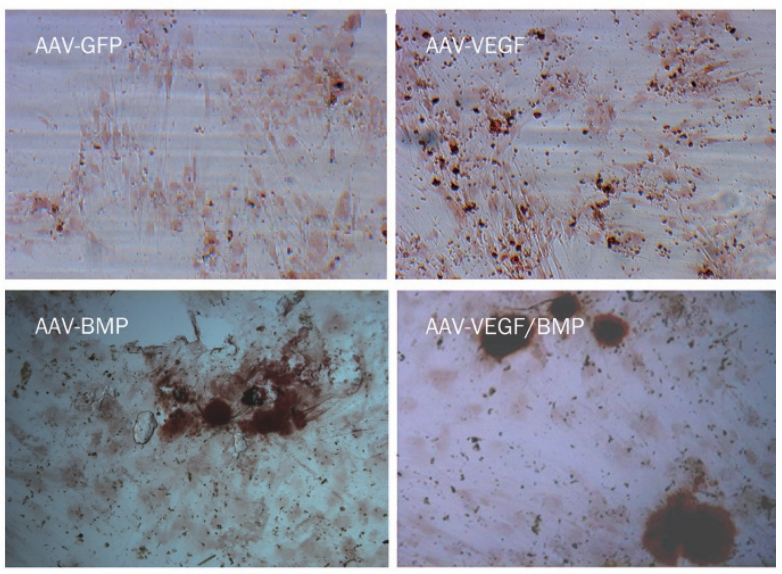

C

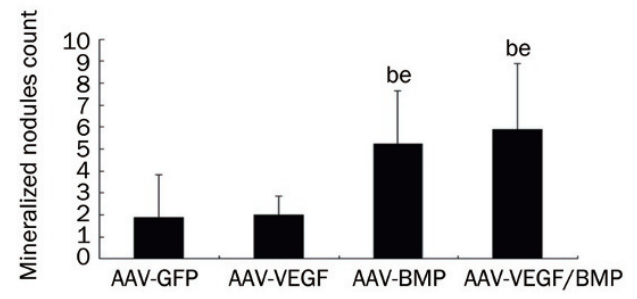

Figure 5. Osteogenic assay with BMSCs. (A) Representative images of von Kossa staining. BMSCs were stained by the von Kossa method and the mineralization is seen as black dots. (B) Representative images of alizarin red staining. BMSCs were stained by the alizarin red method and the mineralization is seen as mineralized nodules. (C) The data is expressed as the mean $\pm S D$ from three independent experiments. (magnification $\times 400$ ). ${ }^{b} P<0.05$ vs AAV-GFP group. ${ }^{e} P<0.05$ vs AAV-VEGF group.

thetase. The time required for this to occur may be several days, weeks, or months and is dependent on the infection surrounding the host cells ${ }^{[20]}$. For this reason, it is essential to detect the timing of gene expression in vitro and in vivo. The results of GFP expression and RT-PCR analysis in vitro and of Western blotting and ELISA assays in vivo revealed expression of the genes of interest, indicating that the rAAV vector has superior gene expressing ability.

The key aim of gene therapy for osteonecrosis disease is bone and vessel regeneration. Bone restoration is a compli- 
A

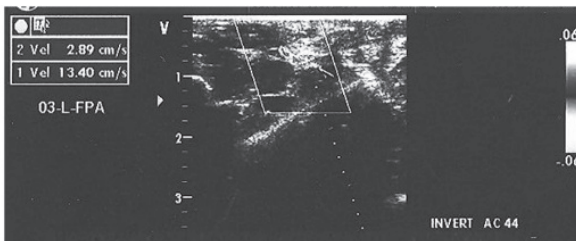

AAV-GFP
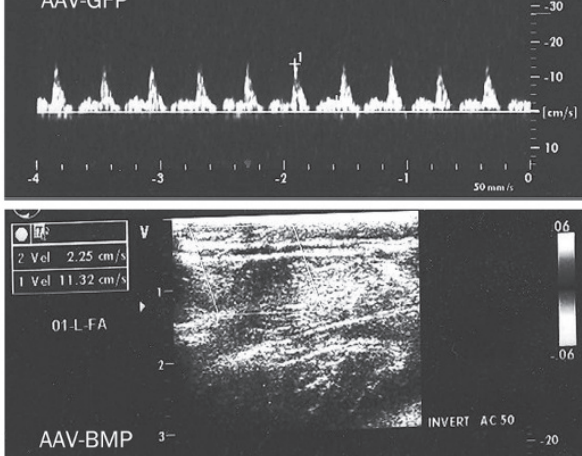

unduntudutul

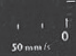

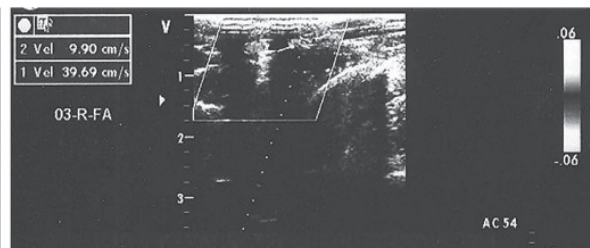

AAV-VEGF
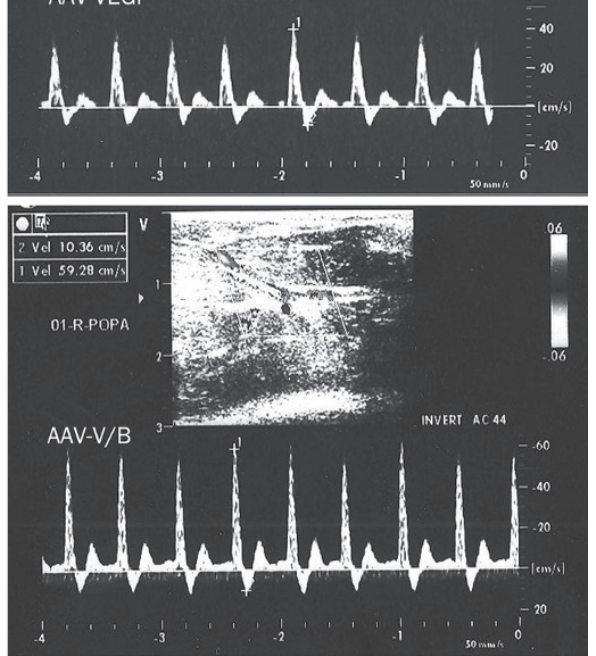

B

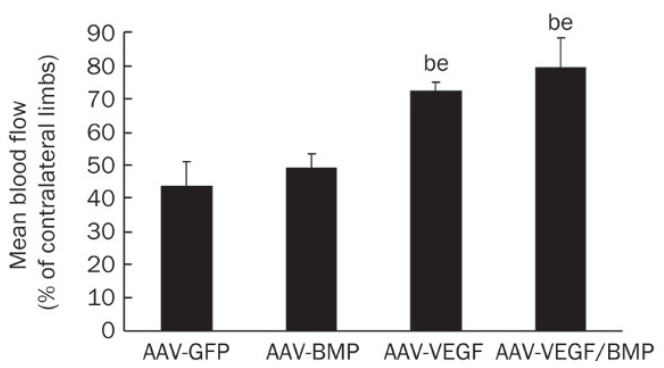

Figure 6. Blood flow in the rabbit hind limb. Blood flow in the anterior tibial artery of ischemic and normal hind limbs was measured at rest with an Aspen Advanced Doppler ultrasound device using a perivascular flow probe 8 weeks post-injection. (A) Representative record images of blood flow in AAV-GFP, AAV-VEGF, and AAV-BMP and AAVVEGF/BMP group. (B) Mean blood flow in the ischemic limbs 8 weeks post-injection. Values are expressed as a percentage of contralateral limbs and are shown as mean values \pm SEM from three independent experiments. ${ }^{\mathrm{b}} P<0.05$ vs AAV-GFP group. ${ }^{\mathrm{e}} P<0.05$ vs AAV-BMP group.
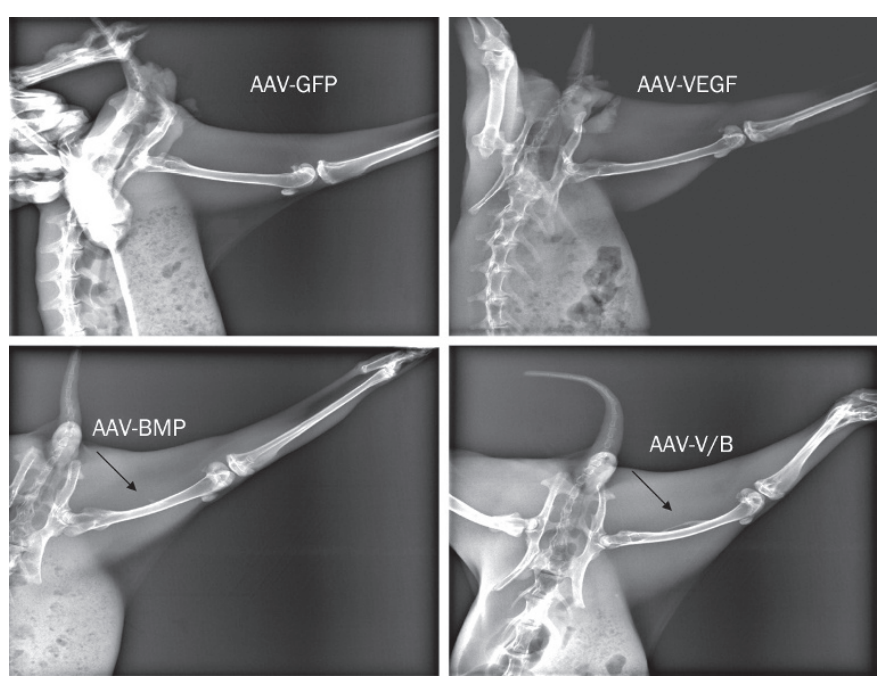

Figure 7. Representative images of orthotopic ossification in the rabbit hind limb. Orthotopic ossification was radiographically evident in AAVVEGF/BMP group eight weeks post injection (indicated as arrows). However, no radiographic evidence of bone formation was observed in AAVGFP group and AAV-VEGF group. cated process involving many kinds of cytokines, and VEGFs and BMPs play important roles during renovation that have been studied extensively. VEGF is one of the most important cytokines in angiogenesis. It specifically promotes the division and growth of vascular endothelial cells and ultimately induces angiopoiesis ${ }^{[2,4,18,20,21]}$. BMPs are the only signaling molecules that can singly induce de novo bone formation at orthotopic and heterotopic sites. BMPs have discrete effects on the proliferation and differentiation of mesenchymal cells and osteoprogenitor cells and also have efficient bone induction activity ${ }^{[3,22]}$. Thus, orchestrating the timing of expression of these two factors may greatly enhance this process.

We performed angiogenic and osteogenic assays to identify the biological effect of $\mathrm{VEGF}_{165}$ and BMP-7 in vitro and in vivo. The results indicated that at the dosage used, the rAAV$\mathrm{hVEGF}_{165}$-IRES-hBMP-7 virus had excellent biological activity and could properly mediate biological activity both in vitro and in vivo. However, one interesting finding of our study was that VEGF alone is not sufficient to improve bone formation and that BMP alone is not sufficient to improve vessel regeneration. We conclude that these findings were not due to improper dosage, but reflect the fact that expression of VEGF 
A
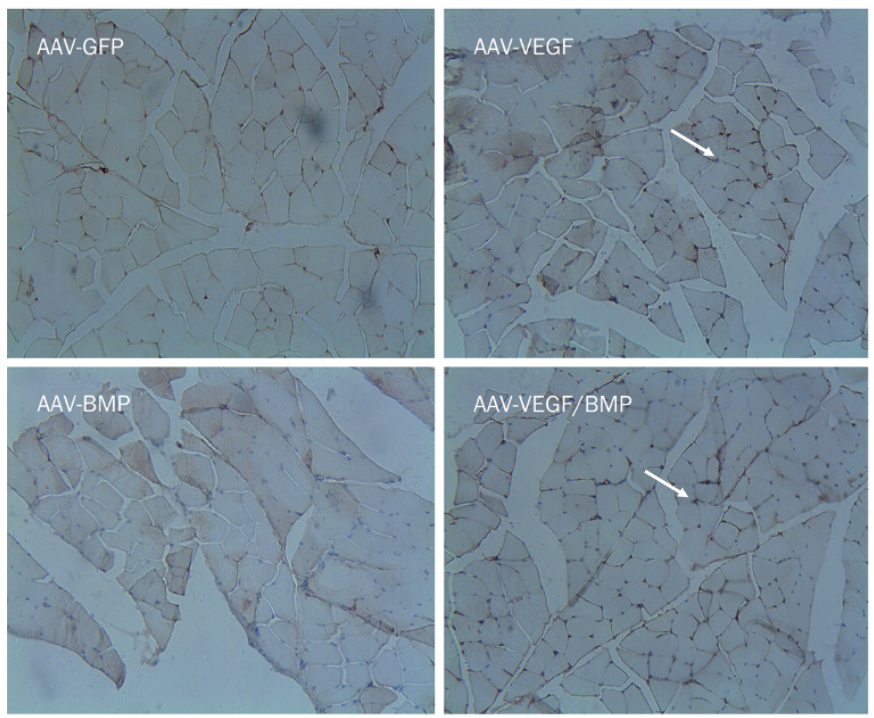

AAV-VEGF/BMP

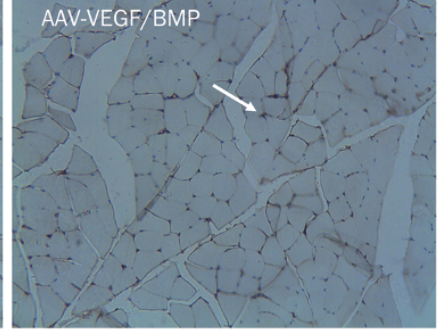

B
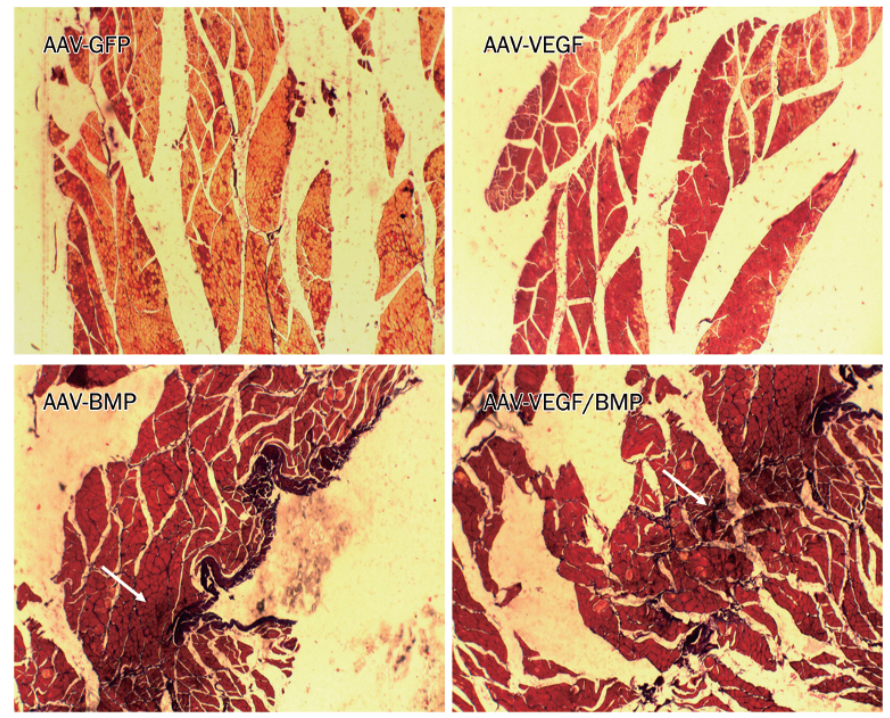

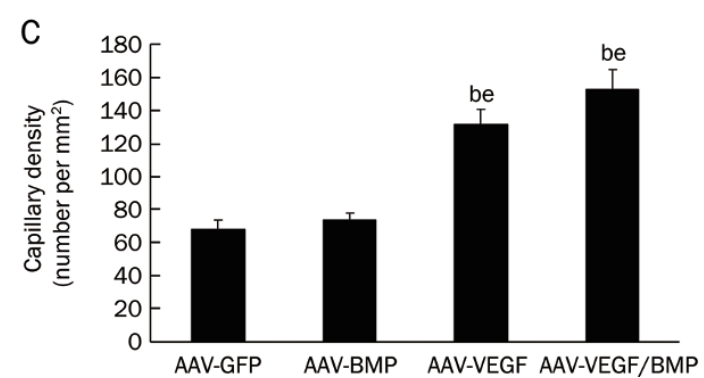

or BMP alone is not sufficient to initiate the cascade of bone formation or vessel regeneration, respectively. These findings also demonstrate that orchestrating the expression of these two factors is essential for effective therapy of osteonecrosis disease. An additional interesting finding in our studies was that there was no statistical difference between the AAV-VEGF/ BMP group and the AAV-VEGF group in terms of angiogenesis. Similarly, there was no statistical difference between the AAV-VEGF/BMP group and the AAV-BMP group in terms of bone formation. We conclude that there may be a requirement for the proper ratio of VEGF to BMP. As shown by a previous study ${ }^{[23]}$, the proper ratio of VEGF to BMP is critical to ensure synergistic effects. In addition, the unequal expression of the VEGF and BMP genes located upstream and downstream of the IRES may be responsible ${ }^{[24]}$. Thus, comparison of the expression of genes located upstream and downstream of the IRES and the identification of the best ratio of VEGF and BMP for treatment of osteonecrosis will be imperative in future experiments.

In summary, we used rAAV as a gene transduction system by successfully inserting the VEGF 165 and BMP-7 genes in this vector, allowing them to be efficiently and stably co-expressed. The VEGF ${ }_{165}$ and BMP-7 proteins that were expressed from the rAAV-hVEGF ${ }_{165}$-IRES-hBMP-7 vector enhanced angiogenesis and bone regeneration in vitro and in vivo. Our experiments establish a foundation for investigating the synergistic biologi- cal effects of $\mathrm{VEGF}_{165}$ and BMP-7 in vitro and in vivo and provide theoretical support for gene therapy of ANFH with our recombinant virus.

\section{Acknowledgements}

This work was supported by the National Natural Science Foundation of China (№ 30600624) and (№ 30772189).

We acknowledge the support of $\mathrm{Xi}^{\prime}$ an Jiao Tong University. We are grateful to Dr Xiang-hui HUANG (Department of Orthopedics, Shaanxi Provincial People's Hospital, Xi'an 710068, Shaanxi Province, China) for his help in the construction of the rAAV-hVEGF 165 -IRES-hBMP-7 viral vector.

\section{Author contribution}

Chen ZHANG designed and performed the experiments; Kunzheng WANG, Hui QIANG, Yi-lun TANG contributed to the in vitro studies; Qian LI and Miao LI contributed to the blood flow measurement studies; Xiao-qian DANG assisted in the design of the study, reviewed all data, and assisted in writing the manuscript. All authors have read and approved the final manuscript.

\section{References}

1 Dai J, Rabie AB. VEGF: an essential mediator of both angiogenesis and endochondral ossification. J Dent Res 2007; 86: 937-50.

2 Clarkin CE, Emery RJ, Pitsillides AA, Wheeler-Jones CP. Evaluation of 
VEGF-mediated signaling in primary human cells reveals a paracrine action for VEGF in osteoblast-mediated crosstalk to endothelial cells. J Cell Physiol 2008; 214: 537-44.

3 Hu J, Qi MC, Zou SJ, Li JH, Luo E. Callus formation enhanced by BMP-7 exvivo gene therapy during distraction osteogenesis in rats. J Orthop Res 2007; 25: 241-51.

4 White AP, Vaccaro AR, Hall JA, Whang PG, Friel BC, McKee MD. Clinical applications of BMP-7/OP-1 in fractures, nonunions and spinal fusion. Int Orthop 2007; 31: 735-41.

5 Hou H, Zhang X, Tang T, Dai K, Ge R. Enhancement of bone formation by genetically-engineered bone marrow stromal cells expressing BMP-2, VEGF and angiopoietin-1. Biotechnol Lett 2009; 31: 1183-9.

6 Young S, Patel ZS, Kretlow JD, Murphy MB, Mountziaris PM, Baggett LS, et al. Dose effect of dual delivery of vascular endothelial growth factor and bone morphogenetic protein- 2 on bone regeneration in a rat critical-size defect model. Tissue Eng Part A 2009; 15: 2347-62.

7 Merten OW, Gény-Fiamma C, Douar AM. Current issues in adenoassociated viral vector production. Gene Ther 2005; 12: S51-61.

8 Grieger JC, Choi VW, Samulski RJ. Production and characterization of adeno-associated viral vectors. Nat Protoc 2006; 1: 1412-28.

9 Tu L, Xu X, Wan H, Zhou C, Deng J, Xu Q, et al. Delivery of recombinant adeno-associated virus-mediated human tissue Kallikrein for therapy of chronic renal failure in rats. Hum Gene Ther 2008; 19: 318-30.

10 Huang X, Shi Z, Wang K, Dang X, Yang P, Yu P. Construction of recombinat adeno-associated virus vector co-expressing hVEGF165 and hBMP-7 genes. Zhongguo Xiu Fu Chong Jian Wai Ke Za Zhi 2008; 22: 807-13. Chinese.

11 Yan H, Guo Y, Zhang P, Zu L, Dong X, Chen L, et al. Superior neovascularization and muscle regeneration in ischemic skeletal muscles following VEGF gene transfer by rAAV1 pseudotyped vectors. Biochem Biophys Res Commun 2005; 336: 287-98.

12 Pittenger MF, Mackay AM, Beck SC, Jaiswal RK, Douglas R, Mosca JD, et al. Multilineage potential of adult human mesenchymal stem cells. Science 1999; 284 (5411): 143-7.

13 Silvestre JS, Tamarat R, Ebrahimian TG, Le-Roux A, Clergue M, Emmanuel $\mathrm{F}$, et al. Vascular endothelial growth factor-B promotes in vivo angiogenesis. Circ Res 2003; 93: 114-23.

14 Byun J, Heard JM, Huh JE, Park SJ, Jung EA, Jeong JO, et al. Efficient expression of the vascular endothelial growth factor gene in vitro and in vivo, using an adeno-associated virus vector. J Mol Cell Cardiol 2001; 33: 295-305.

15 Jaffe EA, Nachman RL, Becker CG, Minick CR. Culture of human endothelial cells derived from umbilical veins: identification by morphologic and immunologic criteria. J Clin Invest 1973; 52 (11): 2745-56.

16 Bellows CG, Aubin JE, Heersche JN, Antosz ME. Mineralized bone nodules formed in vitro from enzymatically released rat calvaria cell populations. Calcif Tissue Int 1986; 38: 143-54.

17 Pelletier J, Sonenberg N. Internal initiation of translation of eukaryotic mRNA directed by a sequence derived from poliovirus RNA. Nature 1998; 334: 320-5.

18 Li W, Thakor N, Xu EY, Huang Y, Chen C, Yu R, et al. An internal ribosomal entry site mediates redox-sensitive translation of Nrf2. Nucleic Acids Res 2010; 38: 778-88.

19 Jeffrey S. Kieft. Viral IRES RNA structures and ribosome interactions. Trends Biochem Sci 2008; 33: 274-83.

20 Tang X, Fu DH, Yang SH, Chen YC, Li Q, Yu CN, et al. Assessment of the expression profile during the entochondrostosis of vascular endothelial growth factor in bone morphogenetic protein 2 induced osteogenesis. Zhonghua Wai Ke Za Zhi 2008; 46: 614-7.

21 Samee M, Kasugai S, Kondo H, Ohya K, Shimokawa H, Kuroda S. Bone morphogenetic protein-2 (BMP-2) and vascular endothelial growth factor (VEGF) transfection to human periosteal cells enhances osteoblast differentiation and bone formation. J Pharmacol Sci 2008; 108: 18-31.

22 Stöve J, Schneider-Wald B, Scharf HP, Schwarz ML. Bone morphogenetic protein 7 (bmp-7) stimulates proteoglycan synthesis in human osteoarthritic chondrocytes in vitro. Biomed Pharmacother 2006; 60: 639-43.

23 Peng H, Wright V, Usas A, Gearhart B, Shen HC, Cummins J, et al. Synergistic enhancement of bone formation and healing by stem cellexpressed VEGF and bone morphogenetic protein-4. J Clin Invest, 2002; 110: 751-9.

24 Kapturczak M, Zolotukhin S, Cross J, Pileggi A, Molano RD, Jorgensen $\mathrm{M}$, et al. Transduction of human and mouse pancreatic islet cells using a bicistronic recombinant adeno-associated viral vector. Mol Ther 2002; 5: 154-60. 